\title{
Wavelet Based Automatic Thresholding for Image Segmentation
}

\author{
Xiao-Ping Zhang and Mita D. Desai \\ Division of Engineering \\ The University of Texas at San Antonio,
}

\begin{abstract}
In this paper, a new systematic method to segment possible target areas based on wavelet transforms is presented. We develop an analytic model for the segmentation of targets, which uses a novel multiresolution analysis in concert with a Bayesian classifier to identify the possible target areas. A method is developed which adaptively chooses thresholds to segment targets from background, by using a multiscale analysis of the image probability density function (PDF). We present examples which demonstrate the efficiency of the technique on a variety of targets.
\end{abstract}

\section{Introduction}

There are many greyscale based segmentation methods, such as thresholding methods [1,2]. Most of these greyscale based segmentation methods often assume that the image has a uniform and stationary or quasistationary distribution of greyscale for various targets or background. So they are often not so effective for the images with complex structure because of the complex distribution of the greyscale of images. Some recent techniques [3] assume images to be mostly nonstationary with space variant distribution. The segmentation methods based on this model are dependent on local area. The performance of such local operator will degrade quickly as the noise increases.

It is reasonable to assume that an image will have complex, nonstationary and space variant greyscale distribution especially when pixel by pixel distributions are considered. However, one can assume that the greyscale distribution will look stationary in the images decomposed at different scales. This is reasonable since, every object has some evident feature in some specific scale space although it may be invisible in other scale space. By wavelet transforms $[4,5]$ and multiscale analysis, the most of the greyscale information can be obtained in large scale image, and the singularity information, such as edge and texture, etc., can be detected in detailed images at different scales. The proposed technique of this paper sets an automatic threshold based on the wavelet analysis of the decomposed image from a wavelet decomposed image of an appropriate scale.

\section{Segmentation of Possible Targets Using Wavelet Transform}

The targets usually appear as blobs in images. It is observed that the global greyscale of the targets is usually higher than that of normal backgrounds. By using wavelet transform and choosing proper mother wavelet, the highfrequency components will disappear in larger scale channels and therefore the blob greyscale will be clearly evident. It is reasonable to assume that in the wavelet transformed images, there are two classes: the background $C_{b}$, and the target $C_{t}$. The segmentation of possible targets then becomes a problem of choosing one of the two hypotheses:

$$
\begin{aligned}
& H_{1}: I(i, j) \in C_{b} \\
& H_{2}: I(i, j) \in C_{t} .
\end{aligned}
$$

Assume $p_{I}(x)$ is the probability distribution function (PDF) of image $I$. Then

$$
p_{I}(x)=P\left(C_{b}\right) p_{b}(x)+P\left(C_{t}\right) p_{t}(x),
$$

where $p_{b}(x)$ is the PDF of the class $C_{b}, P\left(C_{b}\right)$ is the $a$ priori probability of class $C_{b}$ in image $I, p_{t}(x)$ is the PDF

† In Proc. of ICIP'97, Santa Barbara, CA, Oct. 26-29, 1997.

Contact: Dr. X.-P. Zhang, xpzhang@ieee.org, http://www.ee.ryerson.ca/ xzhang 
of the class $C_{t}$, and $P\left(C_{t}\right)$ is the a priori probability of class $C_{t}$ in image.

Therefore, classifiers such as the Bayes classifier and threshold method can be used for classifying into two different types of masses, i.e., normal backgrounds and possible targets:

$$
\left\{\begin{array}{l}
I(i, j)<\lambda: I(i, j) \in C_{b} \\
I(i, j)<\lambda: I(i, j) \in C_{t},
\end{array}\right.
$$

where $\lambda$ is Bayes threshold.

There are two main problems in using the Bayes classifier for image segmentation. First of all, images have a rather complex structure; they contain other classes. For example in a mammogram, the object of segmentation is the tumor which generally has a higher greyscale value than the remainder of the tissue. Within a mammogram, there are calcifications, dense tissue, and various kinds of noise, etc., in addition to the normal tissues and possible tumors (targets). The normal tissues and possible targets also contain some singular pixels. Hence, many pixels may be misclassified by using simple threshold detection method on original mammograms. Secondly, in practice, we do not actually know the PDF of any class as well as the a priori probability of every class. Also, the probability distributions often vary greatly in different images so that we cannot get a general distribution to describe all images.

It is easy to understand that the target usually appear in larger scale channels after the wavelet transform because it usually appear as brighter mass in the image. In the large scale image components $I^{(j)}, j=1,2, \ldots$, the high frequency singularities disappear and the distribution of masses is smoothed. It is more reasonable to assume there are only some mass classes which represent targets and normal background in the transformed image. The normal decimated discrete wavelet transform (DWT) has sampling grid that is very sparse which is desirable for coding and compression applications but often not suitable for signal analysis applications [6]. So the undecimated DWT is used in our method.

By analyzing the image at a coarser scale, singularities can be removed. However, the PDF of any class as well as the a priori probability is still not known in the low resolution images. It is difficult to choose a suitable threshold automatically. Of course, trial and error is a possible approach. However, it is not efficient and difficult to use in practice. We develop a method based on wavelet analysis to find an appropriate threshold adaptively and automatically for different images.

In our method, the threshold is chosen as the "global" local minimum point $\lambda_{m}$, i.e., $\lambda_{m}$ will satisfy the following:

$$
\left.\frac{\partial p_{I}(x)}{\partial x}\right|_{x=\lambda_{m}}=0
$$

and

$$
\left.\frac{\partial^{2} p_{I}(x)}{\partial x^{2}}\right|_{x=\lambda_{m}}>0
$$

This threshold, $\lambda_{m}$, is different from Bayes threshold although they may be very close in many cases. Because the PDFs and the a priori probabilities of the two classes are unknown, and the PDF of every image does not have a one specified distribution, it is impossible to calculate the ideal Bayes threshold. So the proposed new method can usually find a very good threshold and can be reasonably treated as an estimation of the optimal Bayes threshold. After wavelet transforms, the probability distribution of the different classes is likely to approach normal distribution. Based on the assumption of Gaussian distributions, the adaptive threshold by our method can be analyzed. It can be shown that for many cases, the performance of our threshold is often better than conventional threshold methods, such as simple threshold method, etc.

The PDF $p_{I}(x)$ of a given image can be easily computed by the histogram of the entire image. Using a multiscale analysis, we have removed the singularities in the image and make it suitable for target segmentation using a threshold classifier. However, there are usually many fluctuations in the PDFs of the images. In practice, there will be many local minima in PDF curves. Obviously, what we want to find is the "global" local minimum point. In other word, the local minimum point at a large scale. Again, the wavelet transform can be used to analyze the PDF of the image and to find the "global" local minimum point. To get a better localization of the threshold, once again we suggest the use of the undecimated DWT.

In our approach, the wavelet transformed curves of the PDF at every scale are aligned according to the maximum of them. In general, choose the threshold at some scale $s$ of PDF by looking for the largest local minimum. One can also find the local minimum at several scales of PDF, and then get a weighted average as the final threshold for the target segmentation problem.

Next we find the corresponding areas of the segmentation in the original image. Using the selected threshold, we can get the segmented areas $I_{\text {seg }}^{(j)}$, which represent the possible targets, in the wavelet transformed image at scale $j$. Although this area is approximately the position in original image, there will be some spatial shift in the target location in the transformed image compared with the original image because of the time delay $L_{d}$ of the 
FIR wavelet filters. And actually, one pixel in the wavelet transformed image represents a piece of original image, because the image is analyzed by a bank of windows in wavelet transform. The sizes of the windows vary in different scale. We should take this point into account when the segmented areas are mapped to the original image.

Sometimes, this technique results in several large scale "global" local minima of the PDF of images. This is because there exists more than one class of bright blob greyscale areas. In fact, each threshold can separate a kind of bright blob. If only two classes are expected, the largest "global" local minimum should be selected as the threshold for the segmentation of possible target area because it is the most abnormal.

It is also possible to treat the segmented image as a new "original" image, which may include more detail classes. Then the wavelet analysis of PDF can be used once again to classify the different classes inside the segmented area. The process can be iterated until some chosen criterion is satisfied.

\section{Examples}

For example, consider a $512 \times 512$ mammogram image with 4096 greyscale and $100 \mu m \times 100 \mu m /$ pixel, in which there are some different tissues including tumor. The original image is shown as Fig. 1(a). The histogram of original image is given in Fig. 1(b). The wavelet transformed image in scale 2 is used for segmentation. The wavelet analysis of the PDF of the wavelet transformed image in scale 2 is used to select the "global" local minima. Fig. 2(a) and 3(a) show the segmentation results using the selected two largest "global" local minima $\lambda_{1}, \lambda_{2}\left(\lambda_{1}>\lambda_{2}\right)$ as thresholds, respectively. Fig. 2(b), 3(b) show the corresponding masked images, $I_{\text {seg }} \cdot I+I$, which enhance the segmented areas. By using $\lambda_{1}$, we get the tumor area and the bright circle, as shown in Fig. 2. Note that bright circle is just a lead marker used by radiologists. For $\lambda_{2}$, almost all dense tissue areas including spiculations, which are another type of tissue of interest in mammogram, are segmented from the background, as seen in Fig. 3. As we would expect, in this example the wavelet transform of the original image removed the singularities and obtained the mass greyscale information so that the transformed images are more suitable for segmentation. Subsequently, the wavelet transform on the histograms (PDFs) removed the local fluctuations so that we can automatically find the appropriate "global" local minima as adaptive thresholds. And finally, the adaptive thresholds found by our approach segmented the different types of areas of interest. Results of several examples show that our method is effective for various segmentation applications.

\section{Conclusion}

We have presented a new general method for segmentation of targets in an image. Wavelet transforms are used in our method for the segmentation problems of targets in images. An approach for choosing threshold automatically by using wavelet analysis to look for the "global" local minima of the PDF of wavelet transformed images is proposed for general segmentation problems. The examples show that our method is very effective in segmenting different types of bright targets in images.

After our processing, other algorithms, such as boundary based approaches, can also be combined with our method depending on applications. Furthermore, although our method is based on the distribution of the greylevel of image, it is also possible to use the method to other specific features. That is, we can construct a new image by extracting some feature from the original image to form a new image for segmentation.

\section{Acknowledgments}

This work was supported by NIH grant no. GM07717, NASA grant no. NAG10-0155, and Texas Advanced Research program grant no. ARP002-1996.

\section{Reference:}

[1] P. K. Sahoo, S. Soltani, and A. K. C. Wong, "A survey of thresholding techniques," Comput. Vision Graph. Image Processing, vol. 41, pp. 233-260, 1988.

[2] A. K. Jain, Fundamentals of digital image processing, Prentice-Hall, Inc., New Jersy, 1986.

[3] W.-N. Lie, "Automatic target segmentation by locally adaptive image thresholding," IEEE Trans. on Image Processing, vol. 41, no. 7, pp. 1036-1041, July 1995.

[4] S. Mallat, "A theory for multiresolution signal decomposition: the wavelet representation," IEEE Trans. on PAMI., vol. 11, no. 7, pp. 674--693, July 1989.

[5] I. Daubechies, Ten Lectures on Wavelets, Philadelphia:SIAM, 1992.

[6] O. Rioul and P. Duhamel, "Fast algorithms for discrete and continuous wavelet transforms," IEEE Trans. on Info. Theo., vol. 38, no. 2, pp. 569-586, Mar. 1992. 


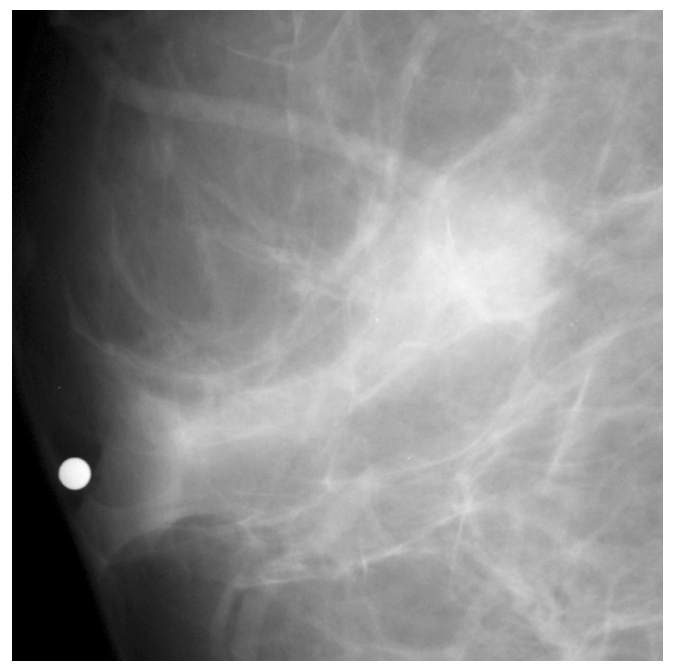

Fig. 1(a) Original mammogram image

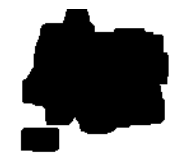

Fig. 2. (a) The segmentation area using $\lambda_{1}$.

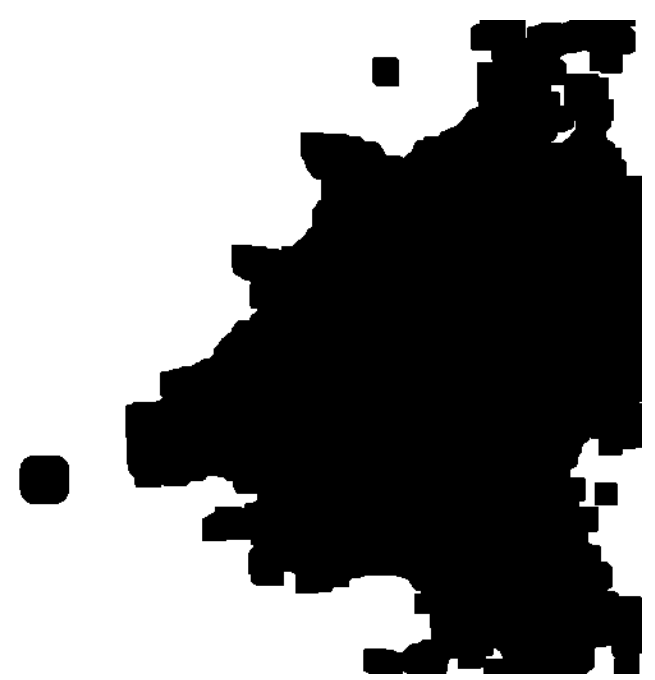

Fig. 3. (a) The segmentation area using $\lambda_{2}$.

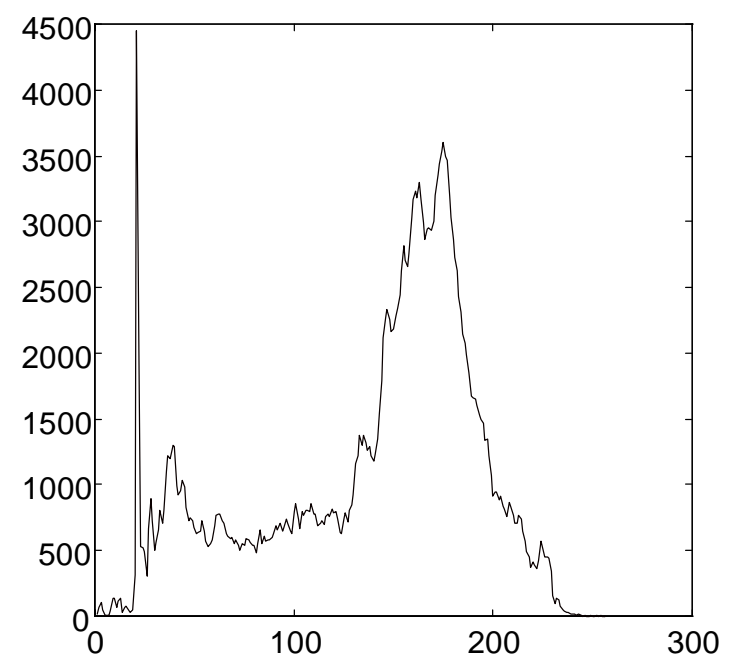

(b) The histogram of the original image

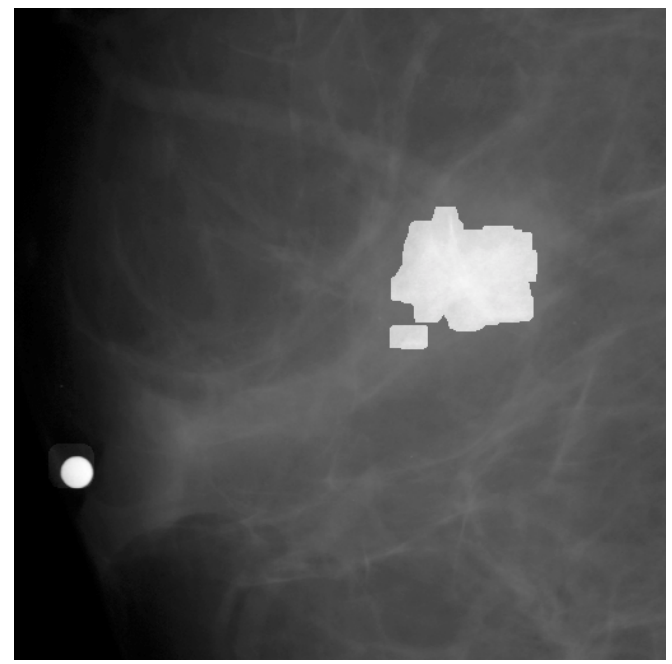

(b) The masked image by the segmentation image in (a).

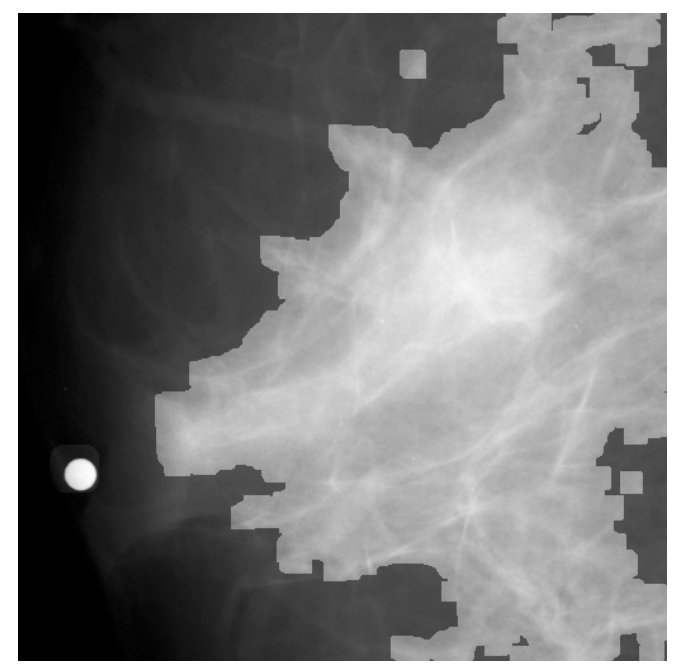

(b) The masked image by the segmentation image in (a). 